Abilene Christian University

Digital Commons @ ACU

$5-13-2020$

Phylogeny and floral character evolution of Mentzelia section Bicuspidaria (Loasaceae)

Joshua M. Brokaw

John J. Schenk

Jessica K. Devitt

Destiny J. Brokaw

Follow this and additional works at: https://digitalcommons.acu.edu/biology

Part of the Biology Commons 
Systematic Botany (2020), 45(2): pp. 306-314

(c) Copyright 2020 by the American Society of Plant Taxonomists

DOI 10.1600/036364420X15862837791258

Date of publication May 13, 2020

\title{
Phylogeny and Floral Character Evolution of Mentzelia Section Bicuspidaria (Loasaceae)
}

\author{
Joshua M. Brokaw, ${ }^{1,4}$ John J. Schenk, ${ }^{2}$ Jessica K. Devitt, ${ }^{3}$ and Destiny J. Brokaw ${ }^{1}$ \\ ${ }^{1}$ Department of Biology, Abilene Christian University, Abilene, Texas 79699-7868, USA; josh.brokaw@acu.edu; djw15a@acu.edu \\ ${ }^{2}$ Department of Environmental and Plant Biology, Ohio University, Athens, Ohio, 45701-2979, USA; schenk@ohio.edu \\ ${ }_{3}^{3}$ Jonah Ventures, Boulder, Colorado 80301, USA; jessicadevitt10@gmail.com. \\ ${ }^{4}$ Author for correspondence (josh.brokaw@acu.edu)
}

\author{
Communicating Editor: Ricarda Riina
}

\begin{abstract}
Mentzelia section Bicuspidaria (Loasaceae) is a monophyletic group of desert ephemerals that inhabit the complex, heterogeneous landscapes of the southwestern United States and northwestern Mexico. To investigate species circumscriptions and evolutionary relationships in $M$. sect. Bicuspidaria, we employed phylogeny reconstructions based on DNA sequences from the plastid trnL-trnF, trnS-trnfM, ndhF-rpl32, and $r p l 32-$ $\operatorname{trnL}$ regions and the nuclear ribosomal ITS and ETS regions. Due to evidence of discordant relationships reconstructed from the plastid and nuclear partitions, we used coalescent-based methods in addition to concatenated data sets to estimate the species tree. Maximum likelihood reconstructions based on the combined plastid and nuclear data and coalescent-based reconstructions inferred congruent, fully-resolved species-level phylogenies of M. sect. Bicuspidaria. A monophyletic M. sect. Bicuspidaria was composed of two main clades, which corresponded to a clade of species endemic to the United States composed of M. reflexa, M. tricuspis, and M. tridentata that was sister to a clade of species at least partially distributed in Mexico, composed of $M$. hirsutissima and $M$. involucrata. Despite the unusual floral morphology of $M$. reflexa, molecular reconstructions placed $M$. reflexa sister to $M$. tridentata. All species of $M$. sect. Bicuspidaria were monophyletic, except for M. hirsutissima, which was composed of two distinct lineages and paraphyletic with respect to $M$. involucrata. The northern clade of $M$. hirsutissima from California and Baja California was sister to $M$. involucrata, and both, in turn, were sister to a geographically disjunct southern clade of M. hirsutissima from Baja California Sur and Cedros Island. These phylogeny reconstructions provide evidence for the inclusion of five species in M. sect. Bicuspidaria and have uncovered cryptic diversity that has been largely unrecognized. Character state reconstructions based on the phylogeny of $M$. sect. Bicuspidaria suggest innovative and, at times, homoplasious floral evolution.
\end{abstract}

Keywords—cpDNA, coalescent model, cryptic species, diversification, floral morphology, homoplasy, nrDNA.

Mentzelia L. section Bicuspidaria S.Watson (Loasaceae) is a monophyletic group composed of five described species, which include $M$. hirsutissima S.Watson, M. involucrata S.Watson, M. reflexa Coville, $M$. tricuspis A.Gray, and $M$. tridentata (Davidson) H.J.Thomps. \& J.E.Roberts (Daniels 1970; Hufford 2003; Hufford et al. 2003; Brokaw 2016). The name of the section refers to the presence of two lateral cusps on the stamen filaments in most of the species. Mentzelia sect. Bicuspidaria is primarily distributed in the southwestern United States and northwestern Mexico. The species are desert ephemerals, usually occurring in dry, coarse-textured soils. It has been suggested that species within $M$. sect. Bicuspidaria require specific combinations of temperature and precipitation for germination and life cycle completion and that these factors correspond to geography (Daniels 1970). Consequently, each species in $M$. sect. Bicuspidaria inhabits a unique geographic area, and sympatric populations have only rarely been observed (Daniels 1970; Brokaw 2016).

Despite M. sect. Bicuspidaria consisting of only five described species, significant questions have been raised regarding the composition of species within it. Previous studies that included most of the taxa within $M$. sect. Bicuspidaria did not resolve all species-level relationships and did not include a thorough sampling of populations that might be needed to understand species boundaries (Hufford et al. 2003; Schenk and Hufford 2011). Different authors have proposed conflicting circumscriptions of the section based on morphology (Urban and Gilg 1900; Darlington 1934; Daniels 1970; Brokaw 2016); however, the homology of morphological characters must be assessed in order to infer evolutionary relationships, and hypotheses of homology have not always been straightforward in Loasaceae (Weigend 1997; Hufford 2003). Morphological comparison and circumscription of individual species within $M$. sect. Bicuspidaria have also been challenging due to high levels of intraspecific variation and low interspecific variation (Daniels 1970). Therefore, it is necessary to reconstruct a well-supported phylogeny using independent molecular markers to test classifications based on morphology while investigating the patterns of morphological evolution in M. sect. Bicuspidaria. A complete species-level phylogeny, however, has never been reconstructed for $M$. sect. Bicuspidaria.

The most notable point of taxonomic confusion in $M$. sect. Bicuspidaria has been interpretation of the relationship between $M$. reflexa and the remaining taxa. In one of the earliest Mentzelia studies, Urban and Gilg (1900) placed M. reflexa into its own section, $M$. sect. Octopetaleia Urb. \& Gilg, in reference to the eight petals on most specimens. Darlington (1934), however, did not recognize $M$. sect. Octopetaleia and placed M. reflexa in M. sect. Bartonia Torr. \& A.Gray. Daniels (1970) was the first to place M. reflexa in M. sect. Bicuspidaria, and his hypothesis has been supported by subsequent molecular analyses (Hufford et al. 2003; Schenk and Hufford 2011).

The primary objective of this study was to reconstruct a fully resolved species-level phylogeny of $M$. sect. Bicuspidaria in order to test sectional and species circumscriptions and to infer patterns of floral evolution. Populations were sampled to reflect relative abundance, range size, and geographic distribution of each species (Appendix 1) in order to resolve ambiguous patterns of intraspecific and interspecific variation that have obscured interpretations of species diversity. We sampled multiple markers from the chloroplast and nuclear genomes to reconstruct a robust species-tree hypothesis that was used to distinguish homologous and homoplasious morphological traits.

\section{Materials ANd Methods}

Taxon Sampling - We sampled 45 herbarium accessions (Appendix 1), representing all five species of Mentzelia section Bicuspidaria recognized by Brokaw (2016). Although the most recent treatment of $M$. sect. Bicuspidaria does not recognize infraspecific taxa (Brokaw 2016), we sampled accessions of $M$. involucrata that keyed to both $M$. involucrata var. involucrata I.M.Johnst and M. involucrata var. megalantha I.M.Johnst according to 
Thompson and Roberts (1974). We have sampled accessions consistent with the varieties of M. hirsutissima described by Johnston (1922), including M. hirsutissima var. hirsutissima I.M.Johnst, Mentzelia hirsutissima var. nesiotes I.M.Johnst, and Mentzelia hirsutissima var. stenophylla (Urb. \& Gilg) I.M.Johnst., but Johnston (1924) determined that these taxa cannot be distinguished morphologically by characters designated in the protologue (see Discussion). We selected 12 outgroup taxa based on the familywide phylogeny of Hufford et al. (2003). The outgroup included four of the remaining five sections of Mentzelia recognized by Hufford et al. (2016; the monotypic M. sect. Dendromentzelia Urb. \& Gilg was not sampled). When available, DNA sequence data for these vouchers were selected from GenBank for molecular analyses. The complete list of vouchers and GenBank accession numbers for all sequences used in this study can be found in Appendix 1.

Laboratory Methods - Total genomic DNA was isolated from approximately $10 \mathrm{mg}$ of leaf material from herbarium specimens using the CTAB procedure of Doyle and Doyle (1987) or an EZNA plant DNA kit (Omega Bio-Tek, Norcross, Georgia) following the manufacturer's instructions. The chloroplast spacers trnL-trnF, trnS-trnfM, ndhF-rpl32, and rpl32-trnL, and the ITS and $5^{\prime}$ external transcribed spacer (ETS) regions of nuclear ribosomal DNA were amplified by PCR as described in Marlowe and Hufford (2007), Brokaw and Hufford (2010b), and Schenk and Hufford (2011). We used the following primers for amplification: $\operatorname{trnL}$-trnF with primers TabC and TabF (Taberlet et al. 1991); trnS-trnfM with primers trnS $^{\text {UGA }}$ and trnfM ${ }^{\mathrm{CAU}}$ (Demesure et al. 1995); ndhF-rpl32 with primers ndhF and rpL32-R (Shaw et al. 2007); rpl32-trnL with primers rpL32-F and trnL ${ }^{\text {(UAG) }}$ (Shaw et al. 2007); ITS with primers N-nc18s10 and C26A (Wen and Zimmer 1996); and ETS with primers ETS-MentF (Schenk and Hufford 2011) and 18S-R (Baldwin and Markos 1998).

The PCR protocol for all amplicons consisted of a $25 \mu \mathrm{L}$ sample containing $13.8 \mu \mathrm{L} \mathrm{H}_{2} \mathrm{O}, 2.5 \mu \mathrm{L} 10 \times$ Thermopol reaction buffer with $20 \mathrm{mM}$ $\mathrm{Mg}^{2+}$ (New England Biolabs, Ipswich, Massachusetts), $2.5 \mu \mathrm{L}$ of each $5 \mathrm{mM}$ primer, $1.5 \mu \mathrm{L} 2.5 \mathrm{mM}$ dNTP, $0.2 \mu \mathrm{L} 5 \mathrm{U} / \mu \mathrm{L}$ Taq polymerase (New England Biolabs), and $2.0 \mu \mathrm{L}$ 1:10 diluted DNA template of unknown concentration. PCR conditions in a Biometra thermocycler (Whatman, Göttingen, Germany) included initial denaturation at $94^{\circ} \mathrm{C}$ for $5 \mathrm{~min}$ followed by 30 cycles at $94^{\circ} \mathrm{C}$ for $1 \mathrm{~min}, 55^{\circ} \mathrm{C}$ for $1 \mathrm{~min}$, and $72^{\circ} \mathrm{C}$ for $2 \mathrm{~min}$; with a final extension at $72^{\circ} \mathrm{C}$ for $7 \mathrm{~min}$. All PCR products were visualized by $1 \%$ agarose gel electrophoresis and purified with an EZNA Cycle-Pure Kit (Omega Bio-Tek) or ExoSAP-IT (Affymetrix, Cleveland, Ohio) following the manufacturers' instructions. The purified products were directly sequenced bidirectionally at the DNA Analysis Facility at Yale University. Sequences were assembled and edited using the program Sequencher v. 5.4.6 (Gene Codes Corp., Ann Arbor, Michigan).

Phylogenetic Analyses-Each DNA sequence region was aligned separately using SATé v. 2.2.7 (Liu et al. 2012) with the MAFFT (Katoh and Standley 2013) aligner and the MUSCLE (Edgar 2004) merger. Trees were generated for the purpose of the alignment with RAxML (Stamatakis 2014) and the GTR + I + G substitution model. We applied the blind mode to allow 20 iterations of tree searches and alignments following the last improvement in likelihood scores; all other settings were set to the SATé defaults. Alignments created by SATé were manually inspected and edited with Mesquite v. 3.31 (Maddison and Maddison 2017) to further reduce homoplasy at ambiguous sites if needed. Following alignment, we selected the best model of nucleotide substitution for each DNA sequence region as well as a concatenated alignment using the Akaike information criterion (AIC) in jModelTest v. 2.1.4 (Darriba et al. 2012).

We used the incongruence length difference (ILD; Farris et al. 1995) test implemented in PAUP* v. 4.0a159 (Swofford 2002) as a partition homogeneity test for topological incongruences within and between the plastid and nuclear data sets. Separate tests were used to examine overall character incongruence among the plastid spacers, between the ITS and ETS regions, and between the plastid and nuclear data sets. Tests for incongruence between the plastid and nuclear data sets were performed with all accessions and with only accessions from M. sects. Bartonia and Bicuspidaria. All analyses applied 1000 test replicates, each with 100 random order entry heuristic searches and one tree saved per replicate. We also applied the guidelines of Nieto Feliner and Rosselloì (2007) to subsequent decisions regarding the combination cpDNA and nrDNA sequences in phylogeny reconstructions.

We conducted maximum likelihood (ML; Felsenstein 1981) analyses using RAxML v. 8.0.22 (Stamatakis 2014) through the CIPRES Science Gateway v. 3.3 (Miller et al. 2010) under the GTR + I + G model. Gaps were treated as missing data, and each DNA sequence region was treated as a separate partition, allowing the GTR model parameters, branch lengths, and alpha parameter of the gamma distribution to be estimated independently for each region. The best ML tree was chosen from the combined results of 1000 replicated searches. We also conducted neighborjoining (NJ; Saitou and Nei 1987) analyses using PAUP* based on TamuraNei genetic distance (Tamura and Nei 1993; Tamura et al. 2004). Separate ML and NJ analyses were performed for the following combinations of accessions and partitions: 1) all accessions with plastid regions, 2) all accessions with nuclear regions, 3) all accessions with all DNA regions, 4) accessions from $M$. sects. Bartonia and Bicuspidaria with plastid regions, 5) accessions from $M$. sects. Bartonia and Bicuspidaria with nuclear regions, and 6) accessions from M. sects. Bartonia and Bicuspidaria with all DNA regions. In order to more thoroughly explore tree space, we performed the final ML analysis with the concatenated data partitions from all accessions and all DNA regions using PAUP*, with tree bisection-reconnection (TBR) branch swapping, 100 random-addition replicates, and no limit to the maximum number of trees compared.

Clade support was assessed for all ML trees with nonparametric bootstrap (BS) proportions estimated by $1000 \mathrm{ML}$ replicated searches in RAxML using search procedures above. Clade support was assessed for all NJ trees with BS proportions estimated by 1000 NJ replicated searches in

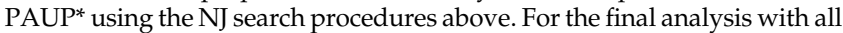
concatenated data partitions, we also estimated Bayesian posterior probabilities (BPP; Huelsenbeck and Ronquist 2001) with MrBayes v. 3.2.2 (Ronquist and Huelsenbeck 2003) through the CIPRES Science Gateway. The Markov chain Monte Carlo chain was run for 30 million generations, and the chain was sampled every 1000 generations after the burn-in period. The burn-in period was determined in Tracer v. 1.6 (Rambaut and Drummond 2005) and convergence was assessed by assuring that the standard deviation of split frequencies was $<0.01$ and by evaluating the trees of each replicated run in the web-based system AWTY (Wilgenbusch et al. 2004; Nylander et al. 2008). The first 10\% of trees were discarded as burn-in.

Discordant relationships within $M$. sect. Bicuspidaria reconstructed from the nuclear and plastid partitions when all accessions were included led us to apply the coalescent species-tree-algorithm implemented in the *BEAST v. 3.0 extension (Heled and Drummond 2010) of BEAST v. 1.8.4 (Drummond et al. 2012) to infer the species tree of M. sect. Bicuspidaria from multilocus data with potentially discordant gene trees (Kubatko and Degnan 2007). Only accessions from M. sects. Bartonia and Bicuspidaria were included. Phylogenetic reconstructions based on the individual gene trees consistently determined that accessions of all named species were monophyletic, except for $M$. hirsutissima. In all reconstructions, $M$. hirsutissima was paraphyletic with respect to $M$. involucrata and separated into a clade of northern accessions ('M. hirsutissima north') and a clade of southern accessions ('M. hirsutissima south'). In only the reconstructions based on the nuclear regions, ' $M$. hirsutissima north' was also paraphyletic with respect to $M$. involucrata and separated into a large clade informally designated as ' $M$. hirsutissima north 1 ' and a small clade designated as ' $M$. hirsutissima north 2.' Therefore, 'M. hirsutissima north $1,{ }^{\prime}$ ' $M$. hirsutissima north 2,' and 'M. hirsutissima south' were designated as putative species for the purpose of species tree estimation with *BEAST. We conducted two runs that sampled every 1000 generations for $1.5 \times 10^{9}$ generations, with the first $10 \%$ of samples discarded as burn-in. The two runs were analyzed for convergence diagnostics with AWTY, and we summarized the data from a single, randomly chosen analysis with a maximum clade credibility tree in TreeAnnotator.

Ancestral Character State Estimations-Using the RAxML methods described above, we reconstructed a phylogeny with branch lengths of selected diploid species from M. sects. Bicuspidaria, Bartonia, and Trachyphytum (Torr. \& A.Gray) Benth. \& Hook.f. based on the chloroplast regions trnL-trnF, trnS-trnfM, ndhF-rpl32, and rpl32-trnL. The following species and lineages were each represented by a single accession (Appendix 1): ' $M$. hirsutissima north,' 'M. hirsutissima south,' $M$. involucrata, $M$. reflexa, M. tricuspis, and M. tridentata from M. sect. Bicuspidaria; M. goodrichii, M. humilis, M. laevicaulis, and M. marginata from M. sect. Bartonia; and $M$. affinis, $M$. congesta, $M$. desertorum, $M$. eremophila, $M$. micrantha, $M$. nitens, M. pectinata, and M. thompsonii from M. sect. Trachyphytum.

Maximum likelihood character state reconstructions were performed for presence or absence of bicuspid stamens and presence or absence of white bracts that directly subtend each flower. We fit two models of character transitions to the data; an equal rates model that allowed for a single rate of transitions between the binary traits, and an all rates different model that allowed for separate transition rates between binary traits. The fit of the models was assessed with AIC values, and we preferred more complex models if they were different by two or greater AIC values (Anderson and Burnham 2002). The best-fit model was then applied to infer the ancestral state at nodes with the APE package (Paradis et al. 2004) in R (R Development Core Team 2005). 


\section{Results}

Phylogenetic Data-The aligned DNA sequences from the combined data partitions resulted in a concatenated alignment that was 5534 base pairs in length. Sequence alignments, XML files for species delimitation analyses in *BEAST, trees obtained from analyses, and supplementary Figs. S1-S7 are available from the Dryad Digital Repository (Brokaw et al. 2019). Plastid and nuclear DNA markers provided similar numbers of informative characters for ML reconstructions; however, the consistency index (CI) of the plastid partition was substantially higher than the CI of the nuclear partition (Table 1).

Congruence of the Data Partitions-Results of the ILD tests for the full set of accessions detected no significant incongruence among plastid spacers or between ITS and ETS partitions ( $p=1.00$ and 1.00, respectively; $\alpha=0.05$ ). The ILD test for the full set of accessions, however, detected significant incongruence between the plastid and nuclear partitions ( $p=$ 0.02). When the ILD test was performed with alignments containing only accessions from $M$. sects. Bartonia and Bicuspidaria, the incongruence between the plastid and nuclear partitions was nonsignificant $(p=0.07)$.

Topologies resulting from separate ML analyses of the plastid and nuclear partitions were incongruent, predominantly in the placement of outgroup lineages, but most incongruent branches (and all incongruent branches connected to outgroups) had low BS support in at least one of the topologies (Fig. S1). The only topological incongruence between plastid and nuclear partitions with strong BS support occurred among accessions of M. hirsutissima: in the plastid ML reconstruction, 'M. hirsutissima north' was monophyletic (Figs. S1A, S3A), whereas, in the nuclear ML reconstruction, ' $M$. hirsutissima north' was paraphyletic with respect to $M$. involucrata (Figs. S1C, S3B).

In contrast, topologies resulting from separate $\mathrm{NJ}$ analyses of the plastid and nuclear partitions had congruent outgroup placement that was congruent with the ML topology based on the plastid partition (Figs. S1, S2). In agreement with the ML topologies, 'M. hirsutissima north' was monophyletic in the plastid NJ reconstruction, whereas ' $M$. hirsutissima north' was paraphyletic with respect to $M$. involucrata in the nuclear NJ reconstruction; all remaining incongruences between the NJ plastid and nuclear topologies and the ML plastid topology occurred among short branches, usually describing intraspecific variation with low BS support (Figs. S1, S2).

When ML analyses were performed with only accessions from $M$. sects. Bartonia and Bicuspidaria, the incongruence associated with 'M. hirsutissima north' persisted, but no other major differences occurred between the plastid and nuclear reconstructions (Fig. S3). The ML and NJ reconstructions were highly congruent when based upon the same data partitions (Figs. S3, S4).
Analyses of the Combined Dataset-All analyses based on the combined plastid and nuclear partitions produced reconstructions with fully resolved interspecific relationships that were congruent with the plastid-only ML and NJ reconstructions (Figs. 1, 2, S1-S7). For all branches describing interspecific relationships, BS support was equal or stronger in the ML and NJ combined reconstructions than for the plastidonly reconstructions. Bayesian posterior probability support was consistent with ML and NJ BS support (Figs. 1, 2, S1-S7). Species in M. sect. Bicuspidaria formed two strongly supported clades, including 1) a clade of species endemic to the United States containing $M$. tricuspis, $M$. tridentata, and $M$. reflexa that we refer to informally as 'Tricuspes' and 2) a clade of species at least partially distributed in Mexico containing M. hirsutissima and $M$. involucrata that we refer to informally as 'Hirsutissimae' (Fig. 2). Although BS support for the 'Tricuspes' and 'Hirsutissimae' clades was already greater than $80 \%$ in the plastid-only reconstructions, BS support for both was increased to $100 \%$ in the combined analysis. In the 'Tricuspes' clade of $M$. sect. Bicuspidaria, accessions from each of the three species included populations that were placed in strongly supported monophyletic groups consistent with the species designations, and the clade of $M$. reflexa accessions was sister to the clade of $M$. tridentata accessions. In the 'Hirsutissimae' clade of $M$. sect. Bicuspidaria, accessions from $M$. involucrata were placed in a strongly supported monophyletic group, but reconstructions did not support the monophyly of the infraspecific taxa $M$. involucrata var. involucrata and $M$. involucrata var. megalantha (Fig. 2). In contrast, accessions representing M. hirsutissima were paraphyletic with respect to $M$. involucrata. A strongly supported clade of ' $M$. hirsutissima north' accessions was placed sister to the clade of $M$. involucrata accessions with strong BS support. A strongly supported clade of ' $M$. hirsutissima south' accessions was placed sister to the clade containing M. involucrata and 'M. hirsutissima north.'

The topology recovered from the *Beast species tree analysis based on plastid and nuclear partitions had strong BPP support and was congruent with other analyses based on the combined plastid and nuclear partitions (Figs. 1, 2, S5-S7). The two putative clades of ' $M$. hirsutissima north' ('M. hirsutissima north 1 ' and ' $M$. hirsutissima north 2 ') that were designated as separate species in *Beast in order to test patterns of incongruence between the plastid and nuclear partitions formed a monophyletic group in the *Beast reconstruction and all other combined analyses (Figs. 1, 2, S5-S7). In agreement with the *Beast reconstruction, ML and NJ analyses of the combined plastid and nuclear partitions recovered the separate clades of ' $M$. hirsutissima north 1 ' and ' $M$. hirsutissima north 2 ' accessions and placed them sister to each other in a strongly supported monophyletic 'M. hirsutissima north' (Figs. 1, 2, S5-S7).

TABLE 1. Statistics for datasets and results from ML searches.

\begin{tabular}{|c|c|c|c|c|c|c|}
\hline & \multicolumn{3}{|c|}{ All accessions } & \multicolumn{3}{|c|}{ M. sects. Bartonia and Bicuspidaria } \\
\hline & Plastid & Nuclear & Combined & Plastid & Nuclear & Combined \\
\hline Number of terminal taxa & 57 & 57 & 57 & 49 & 49 & 49 \\
\hline Total number of characters & 4454 & 1080 & 5534 & 4211 & 1069 & 5280 \\
\hline Variable characters & 459 & 325 & 784 & 159 & 165 & 324 \\
\hline Informative characters & 283 & 258 & 541 & 111 & 134 & 245 \\
\hline Consistency index & 0.913 & 0.695 & 0.804 & 0.970 & 0.771 & 0.844 \\
\hline Retention index & 0.955 & 0.890 & 0.918 & 0.991 & 0.953 & 0.963 \\
\hline
\end{tabular}




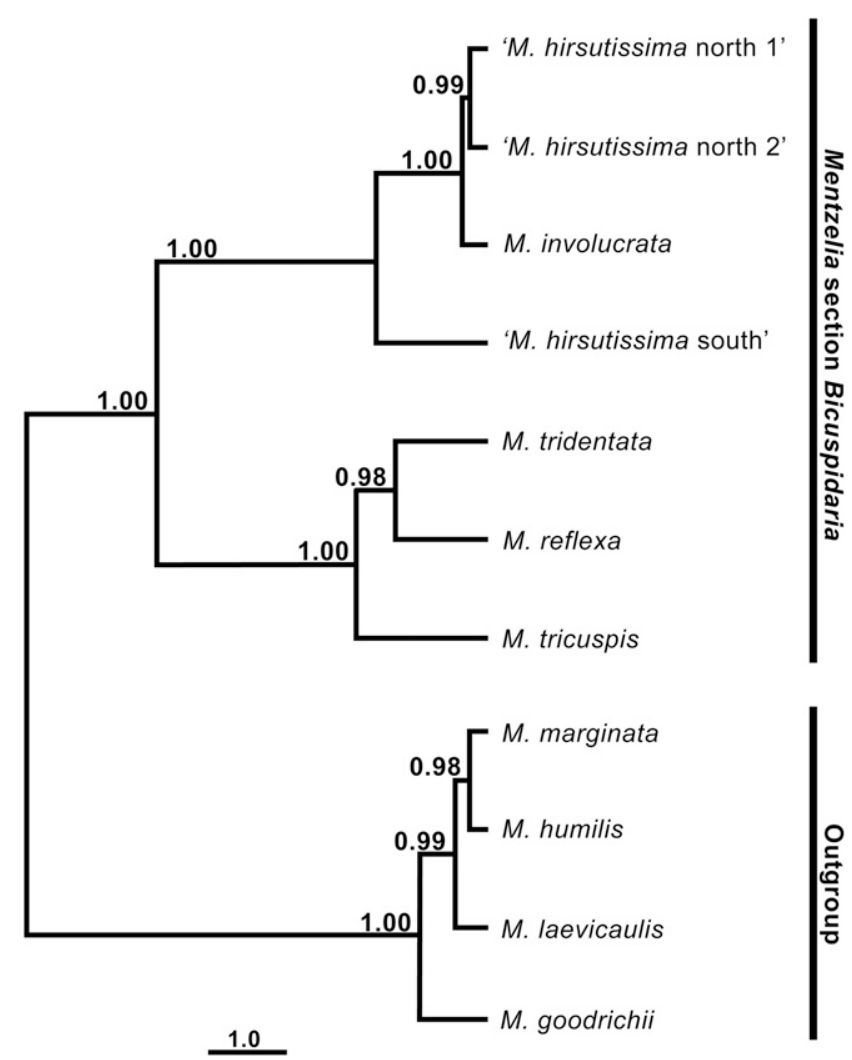

FIG. 1. Bayesian coalescent analysis (*BEAST) based on the combined plastid and nuclear data sets representing only M. sects. Bicuspidaria and Bartonia; values at nodes represent posterior probabilities. Scale bar $=$ expected substitutions per site.

Ancestral Character State Estimations-A model fitting approach to evaluate transition models of binary traits identified the simpler equal rates model as best-fit for the bicuspidate character $(\triangle \mathrm{AIC}=1.170)$, and the all rates different model as best-fit for the bract color $(\Delta \mathrm{AIC}=2.224)$. ML ancestral state reconstructions supported the presence of bicuspidate stamens and green bracts at the most recent common ancestor of M. sect. Bicuspidaria (Fig. 3), suggesting that the unlobed stamens of $M$. reflexa and white bracts of M. involucrata are derived characters. The bicuspidate stamen state at the root of Mentzelia was less certain in the reconstructions; however, bicuspid stamens were most likely absent in the most recent common ancestor of $M$. sect. Trachyphytum (Fig. 3), suggesting that the presence of bicuspid stamens in M. micrantha is derived and homoplasious to $M$. sect. Bicuspidaria. Ancestral state estimations supported the presence of green bracts at the most recent common ancestor of $M$. sect. Trachyphytum (Fig. 3), suggesting that the occurrences of white bracts in $M$. congesta and $M$. involucrata are derived and convergent.

\section{Discussion}

Phylogenetic Reconstruction-This study confirmed that Mentzelia section Bicuspidaria is a monophyletic group, which consisted of interspecific relationships that were fully resolved and strongly supported, with the exception of M. hirsutissima. Although these conclusions are evident with chloroplast markers alone, species tree inferences should be tested for gene tree incongruence when independent sources of data are available (Pamilo and Nei 1988). Thus, we have incorporated ITS and ETS sequences in order to test phylogenetic signal from the nuclear genome and to assess the utility of these markers for phylogenetic studies in Mentzelia.

Problems with phylogenetic reconstruction based on nrDNA are widely recognized (Álvarez and Wendel 2003), but ITS and ETS sequences can still provide insights for estimates of organismal phylogeny when compared to reconstructions based on other markers (Nieto Feliner and Rosselloì 2007). The ILD tests suggested that nrDNA and cpDNA exhibited greatest incongruence when all accessions of the outgroup were included, and ML reconstructions based on nrDNA resulted in poorly supported outgroup placement (Fig. S1C, S1D). In contrast, analyses of nrDNA with the distance-based NJ method recovered exactly the same outgroup topology as the cpDNA ML reconstructions (Fig. S1B), suggesting that nrDNA might be too variable for cladistic reconstruction of the earliest lineages in Mentzelia when used alone. Similar difficulty in root placement with nrDNA has been found in the closely related Loasoideae (Acuña et al. 2017) and Hydrangeeae (De Smet et al. 2015). Following Nieto Feliner and Rosselloì (2007), we combined the cpDNA and nrDNA data sets in a total-evidence ML analysis, and the resulting tree was congruent with the cpDNA ML topology with higher clade support (Figs. 2, S3).

An additional pattern of incongruence between cpDNA and nrDNA topologies occurred in the placement of $M$. hirsutissima. Both plastid and nuclear reconstructions determined that M. hirsutissima s. 1. is paraphyletic (Fig. S3). However, accessions of ' $M$. hirsutissima north' form a monophyletic group in cpDNA reconstructions, whereas nrDNA reconstructions suggested that ' $M$. hirsutissima north' is also paraphyletic with respect to $M$. involucrata. The nrDNA pattern of incongruence could be caused by incomplete lineage sorting (Nieto Feliner and Rosselloì 2007) and was addressed with the coalescent model incorporated into species tree reconstruction with *Beast (Heled and Drummond 2010). The resulting *Beast species tree strongly supported the monophyly of ' $M$. hirsutissima north' in agreement with the total-evidence topology (Figs. 1, 2), even while the two 'M. hirsutissima north' clades recovered with nrDNA data were conservatively treated as different species (Fig. 1).

Cryptic Lineages-Taxonomists have repeatedly struggled to recognize patterns of interspecific variation in $M$. sect. Bicuspidaria on the basis of morphology alone (Johnston 1922, 1924; Darlington 1934; Daniels 1970; Brokaw 2016). The geographically-linked paraphyly of $M$. hirsutissima detected by our molecular analyses provides evidence of a previously unrecognized cryptic lineage.

In similar fashion, the morphologically similar species $M$. tricuspis and $M$. tridentata are most easily distinguished by a geographic disjunction. Mentzelia tridentata was originally described as Acrolasia tridentata by Davidson (1910) and subsequently treated as a synonym of $M$. tricuspis var. brevicornuta I.M.Johnst. by Darlington (1934) before being finally elevated to specific status by Thompson and Roberts (1971). Thompson and Roberts (1971) determined that seed shape and the length of the lateral cusps of the stamens, as first noted by Johnston (1922), consistently differed between the two lineages to provide morphological distinction. Nevertheless, the morphological similarities between these two taxa would seem initially to suggest that they could be at least sister species when, in fact, reconstructions based on cpDNA and nrDNA 


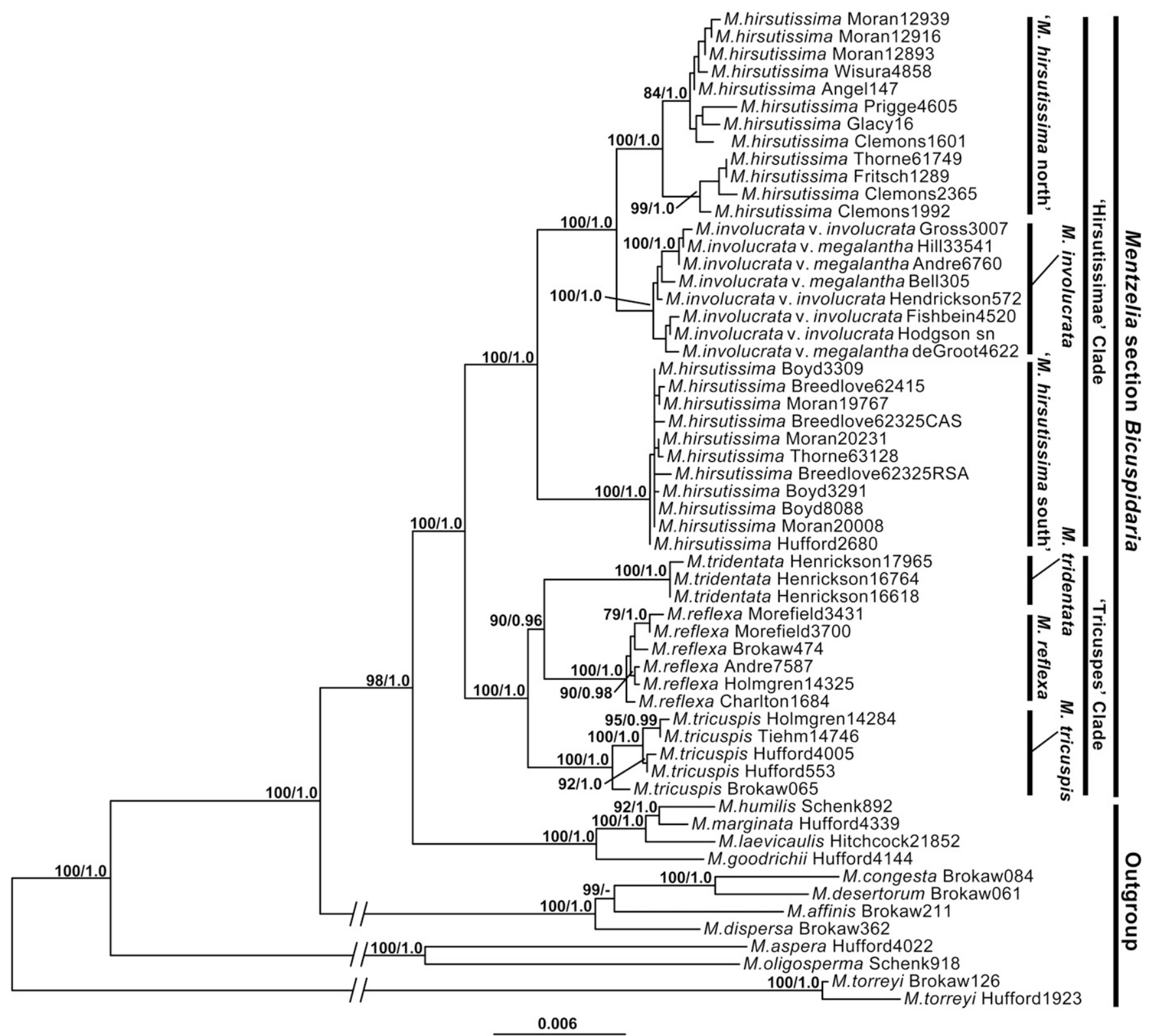

FIG. 2. Maximum likelihood phylogram of Mentzelia section Bicuspidaria and outgroups based on the combined plastid and nuclear data sets; values at nodes represent ML bootstrap proportions $>70 \%$ /Bayesian inference posterior probabilities $>0.95$. Scale bar $=$ expected substitutions per site.

concur that M. tridentata is more closely related to M. reflexa than $M$. tricuspis. Thus, comparisons in a phylogenetic context suggest that the floral characteristics shared by M. tridentata and $M$. tricuspis distinguishing both from $M$. reflexa are symplesiomorphic.

As with M. tricuspis, Johnston (1922) recognized different varieties within $M$. hirsutissima and $M$. involucrata. Daniels (1970) did not recognize subspecific taxa within either species due to the inconsistency of morphological characters used in keys and the incomplete recognition of geographical patterns. In agreement with Daniels (1970), infraspecific phylogenetic patterns among accessions of $M$. involucrata in this study were poorly supported and did not correspond to formerly recognized varieties (Fig. 2).

Nevertheless, the 'M. hirsutissima south' and 'M. hirsutissima north' clades do correspond roughly to 1) M. hirsutissima S.Watson var. nesiotes and 2) $M$. hirsutissima var. hirsutissima plus
M. hirsutissima var. stenophylla respectively (Johnston 1922), but Johnston (1924) had conceded that the primary morphological characters used to distinguish the varieties (flower color and stamen shape) were inconsistent and did not provide alternative characters. Molecular analyses in this study suggested that this diversity in $M$. hirsutissima s. 1 . has been obscured by our focus on morphological symplesiomorphies. Both cpDNA and nrDNA placed ' $M$. hirsutissima north' sister to $M$. involucrata and ' $M$. hirsutissima south' in a more distantly related lineage, suggesting that the paraphyly of $M$. hirsutissima s. 1. is not an artefact of incomplete lineage sorting. Furthermore, this study found no phylogenetic evidence of hybridization among species within $M$. sect. Bicuspidaria, but a similar study of $M$. sect. Trachyphytum (Brokaw and Hufford 2010a, 2010b) detected a substantial history of homoploid hybridization and chloroplast capture. Whereas previous treatments have gone no further than segregating 


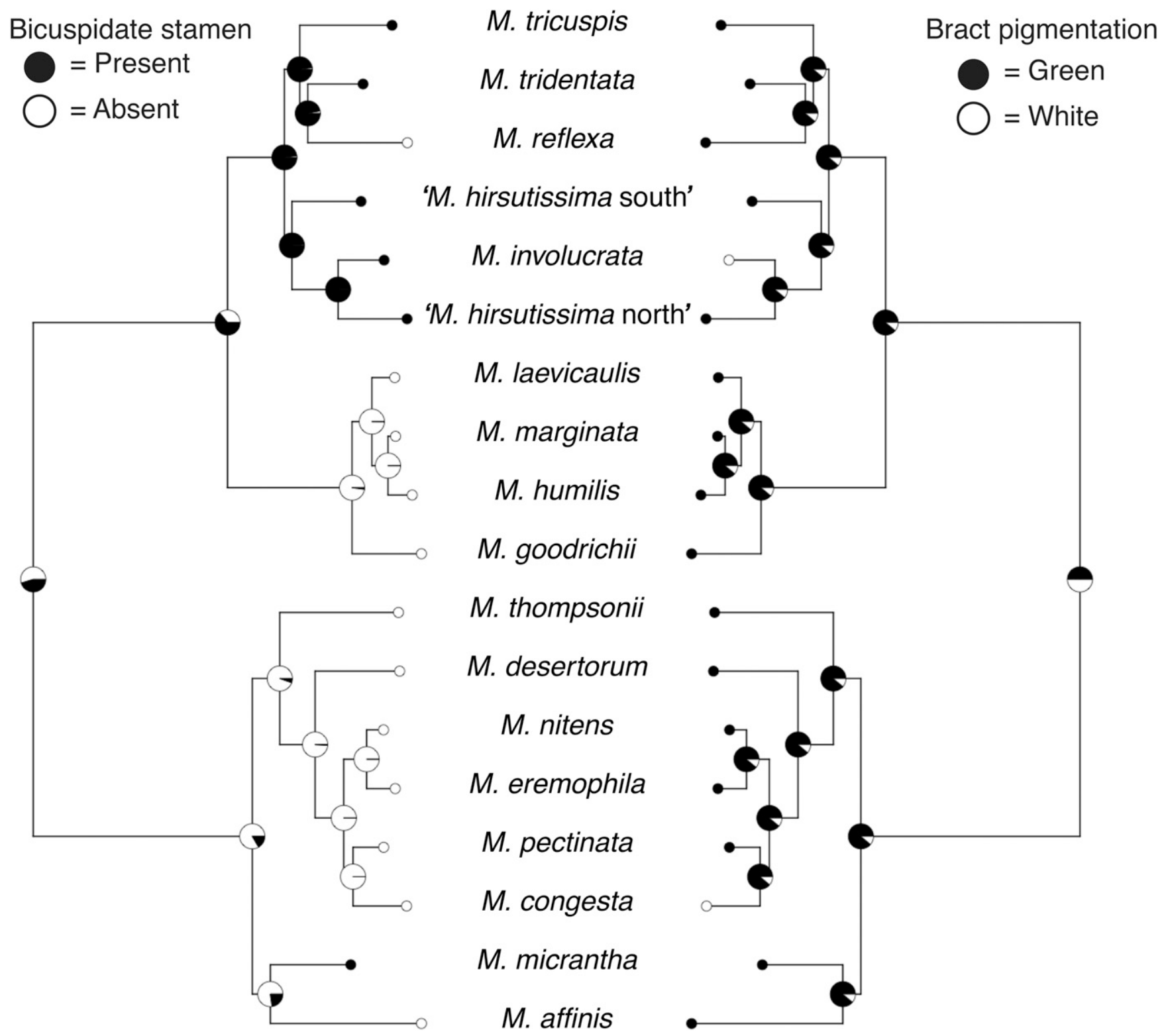

FIG. 3. Ancestral character estimations of bicuspidate stamens and floral bract color in Mentzelia optimized with maximum likelihood.

varieties of M. hirsutissima s. 1. (Johnston 1922; Darlington 1934), this study suggests that ' $M$. hirsutissima north' is more closely related to $M$. involucrata than to 'M. hirsutissima south' and that the shared floral characters distinguishing M. hirsutissima s. 1. from M. involucrata are symplesiomorphies.

Although the non-monophyly of $M$. hirsutissima was not previously suspected, numerous plant and animal species have been shown to exhibit north-south genetic breaks along the Baja California Peninsula (e.g. Upton and Murphy 1997; Riddle et al. 2000; Nason et al. 2002; Lindell et al. 2005; Crews and Hedin 2006; Lira-Noriega et al. 2015). Both M. involucrata and 'M. hirsutissima north' inhabit southern California and the northern part of the Baja California Peninsula with marginally overlapping distributions, whereas the distribution of ' $M$. hirsutissima south' is entirely disjunct from $M$. involucrata and 'M. hirsutissima north' and primarily limited to Baja California Sur. Recent studies have suggested that this pattern could have been caused by vicariance via a midpeninsular seaway in the Miocene or by precipitation gradients preventing migration across the midpeninsular valley (Dolby et al. 2015). In either case, low elevations in the Vizcaíno Plain northeast of ' $M$. hirsutissima south' could have separated these populations from what would later become the 'M. hirsutissima north' and M. involucrata lineages. Significantly, Dolby et al. (2015) noted that reports of such genetic discontinuities have suffered from taxonomic biases and that additional studies reporting the pattern in plant taxa were needed.

The strict adherence of the ' $M$. hirsutissima north' and ' $M$. hirsutissima south' lineages to their respective geographic distributions suggests that physiological adaptations to climate could be used to distinguish them. Topographical dispersal barriers do not seem to be a sufficient explanation for the geographic separation of such old lineages (Schenk 2013), and edaphic factors limiting distributions have not been identified in $M$. sect. Bicuspidaria. However, biologically significant climate factors do appear to distinguish the range of ' $M$. hirsutissima south' from those of ' $M$. hirsutissima north' and M. involucrata. Both 'M. hirsutissima north' and M. involucrata 
occur primarily in eastern deserts that are separated from the Pacific Ocean by mountain ranges, whereas ' $M$. hirsutissima south' inhabits the climatically and vegetationally distinct Vizcaíno fog desert west of the Peninsular Ranges. Although the ecological differences between these species appear to be substantial, we continue to investigate patterns of morphological variation in $M$. hirsutissima s. 1 . in order to update the taxonomy and provide complete species circumscriptions for the ' $M$. hirsutissima north' and ' $M$. hirsutissima south' lineages.

Character Evolution-In general, phylogenetic reconstructions suggest that cladogenesis within $M$. sect. Bicuspidaria has been geographically and morphologically conservative, but a few cases of morphological homoplasy stand out. The morphological affinity of $M$. sects. Bicuspidaria and Bartonia has long been recognized (Brewer and Watson 1876), but species in $M$. sect. Bicuspidaria also share homoplasious floral traits with species in M. sect. Trachyphytum. For example, Hufford (2003) used parsimony-based methods to conclude that the bicuspid stamens present in all species of $M$. sect. Bicuspidaria except $M$. reflexa have a separate origin from the bicuspid stamens of M. micrantha Torr. \& A.Gray in M. sect. Trachyphytum, and our ML reconstructions based on expanded taxon sampling concur. Our reconstructions also strongly suggested that the white scarious bracts of $M$. involucrata have an independent origin from nearly identical bracts in $M$. congesta Torr. \& A.Gray and related polyploids in $M$. sect. Trachyphytum.

Past taxonomic treatments of $M$. reflexa have been especially inconsistent because they have emphasized stamen and staminode morphology (Urban and Gilg 1900; Johnston 1922; Darlington 1934), whereas Botnaru and Schenk (2019) have shown that transitions between stamens and staminodes are common and reversible in $M$. sect. Bartonia. In contrast to other species in M. sect. Bicuspidaria, M. reflexa has a reduced corolla and androecium composed of five narrow petals and one to five (but usually three) petaloid staminodes plus several fertile stamens lacking bicuspid filaments (Brokaw 2016). Floral characters led Urban and Gilg (1900) to place M. reflexa in its own section (M. sect. Octopetaleia) and presumably led Darlington (1934) to place it in $M$. sect. Bartonia, although Thompson (1963) suggested that Darlington's treatment must have inadvertently ignored important seed morphology and life history characters in order to do so. Johnston (1922), when comparing $M$. reflexa to $M$. tricuspis and $M$. tridentata, used floral characters to assert that $M$. reflexa was not closely related to the latter species. However, the phylogeny presented in this study strongly supports an origin of $M$. reflexa from within $M$. sect. Bicuspidaria in the same subclade as M. tricuspis and $M$. tridentata, and our reconstruction of stamen morphology suggested that bicuspid stamens have been lost by M. reflexa. Daniels (1970) was the first to place $M$. reflexa in $M$. sect. Bicuspidaria and based the decision on the general growth habit and leaf, capsule, and seed shapes rather than floral traits. Furthermore, $M$. tridentata is the closest relative to $M$. reflexa in our phylogeny (Fig. 2) and is also geographically the closest to $M$. reflexa among species in $M$. sect. Bicuspidaria, suggesting that habitat requirements of $M$. reflexa and $M$. tridentata have been substantially conserved during cladogenesis. The plesiomorphic flower type in $M$. sect. Bicuspidaria likely has five broad petals with bicuspid stamens but lacks petaloid staminodes (Hufford 2003), suggesting that the absence of bicuspid stamens and presence of petaloid staminodes are derived states in M. reflexa with independent origins from similar traits in M. sect. Bartonia (Botnaru and Schenk 2019).
We are not aware of straightforward adaptive explanations for homoplasy of bicuspid stamen and white bracts in $M$. sect. Bicuspidaria. It is possible that the selective conditions (e.g. pollinators) that produced homoplasious traits are no longer present. Alternatively, some changes such as the reduced petals and modified stamens in $M$. reflexa could be products of relaxed selection and genetic drift in small, self-fertilizing populations rather than adaptation to pollinators. More generally, the phylogenetic distributions of these characters suggest that vegetative characteristics and habitat preferences have been more conserved than floral characteristics within $M$. sect. Bicuspidaria and represent synapomorphies that can be used to distinguish $M$. sect. Bicuspidaria from its closest relatives.

\section{ACKNOWLEDGMENTS}

We thank ACU, CAS, LA, SD, UCR, RSA, and WS for providing material for DNA sequencing; the Rancho Santa Ana Botanic Garden for the use of laboratory facilities; H. J. Thompson, Barry Prigge, Muriel Poston, Dylan Cohen, Loraine Washburn, Rebecca Hunter, Jennifer Huddleston and Qiang $\mathrm{Xu}$ for helpful discussions, technical assistance, and support; and many undergraduate research assistants at Abilene Christian University, including Dylan Almogabar, Hannah Seah, Abigail Rogers, Angela Adhikari, and Tina Johnson for laboratory assistance. This project was funded in part by an Abilene Christian University Math-Science Grant and the Betty W. Higinbotham Trust from Washington State University.

\section{Author Contributions}

JMB and DJB examined herbarium specimens and performed tissue sampling; all authors performed molecular lab work and analyses; JJS, JMB, and JKD performed phylogenetic analyses. All authors shared in writing the manuscript.

\section{Literature Cited}

Anderson, D. R. and K. P. Burnham. 2002. Avoiding pitfalls when using information-theoretic methods. The Journal of Wildlife Management 66: 912-918.

Acuña, R., S. Fließwasser, M. Ackermann, T. Henning, F. Luebert, and M. Weigend. 2017. Phylogenetic relationships and generic rearrangements in "South Andean Loasas" (Loasaceae). Taxon 66: 365-378.

Álvarez, I. and J. F. Wendel. 2003. Ribosomal ITS sequences and plant phylogenetic inference. Molecular Phylogenetics and Evolution 29: 417-434.

Baldwin, B. G. and S. Markos. 1998. Phylogenetic utility of the external transcribed spacer (ETS) of 18S-26S rDNA: Congruence of ETS and ITS trees of Calycadenia (Compositae). Molecular Phylogenetics and Evolution 10: 449-463.

Brewer, W. H. and S. Watson. 1876. Polypetalae. Pp. 1-276 in Botany of California, vol. 1, ed. S. Watson. Cambridge, UK: Welch, Bigelow and Co.

Botnaru, L. and J. J. Schenk. 2019. Staminode evolution in Mentzelia section Bartonia (Loasaceae) and its impact on insect visitation rates. Botanical Journal of the Linnean Society 190: 151-164.

Brokaw, J. M. 2016. Mentzelia section Bicuspidaria. Pp. 524-527 in Flora of North America North of Mexico, vol. 12, ed. Flora North America Editorial Committee. New York: Oxford University Press.

Brokaw, J. M. and L. Hufford. 2010a. Origins and introgression of polyploid species in Mentzelia section Trachyphytum (Losaceae). American Journal of Botany 97: 1457-1473.

Brokaw, J. M. and L. Hufford. 2010b. Phylogeny, introgression, and character evolution of diploid species in Mentzelia section Trachyphytum (Loasaceae). Systematic Botany 35: 601-617.

Brokaw, J. M., J. J. Schenk, J. K. Devitt, and D. J. Brokaw. 2019. Data from: Phylogeny and floral character evolution of Mentzelia section Bicuspidaria (Loasaceae). Dryad Digital Repository. https://doi.org/ 10.5061 /dryad.8v6j202.

Crews, S. C. and M. Hedin. 2006. Studies of morphological and molecular phylogenetic divergence in spiders (Araneae: Homalonychus) from the American Southwest, including divergence along the Baja California peninsula. Molecular Phylogenetics and Evolution 38: 470-487. 
Daniels, G. S. 1970. The Floral Biology and Taxonomy of Mentzelia section Bicuspidaria (Loasaceae). Ph.D. dissertation. Los Angeles: University of California.

Darlington, J. 1934. A monograph of the genus Mentzelia. Annals of the Missouri Botanical Garden 21: 103-227.

Darriba, D., G. L. Taboada, R. Doallo, and D. Posada. 2012. jModelTest 2: More models, new heuristics and parallel computing. Nature Methods 9: 772.

Davidson, A. 1910. Acrolasia tridentata. Bulletin of the Southern California Academy of Sciences 9: 71.

De Smet, Y., C. Granados-Mendoza, S. Wanke, P. Goetghebeur, and M.-S. Samain. 2015. Molecular phylogenetics and new (infra) generic classification to alleviate polyphyly in tribe Hydrangeeae (Cornales: Hydrangeaceae). Taxon 64: 741-753.

Demesure, B., N. Sodzi, and R. J. Petit. 1995. A set of universal primers for amplification of polymorphic non-coding regions of mitochondrial and chloroplast DNA in plants. Molecular Ecology 4: 129-131.

Dolby, G. A., S. E. K. Bennett, A. Lira-Noriega, B. T. Wilder, and A. Munguía-Vega. 2015. Assessing the geological and climatic forcing of biodiversity and evolution surrounding the Gulf of California. Journal of the Southwest 57: 391-455.

Doyle, J. J. and J. L. Doyle. 1987. A rapid DNA isolation procedure for small quantities of fresh leaf tissue. Phytochemical Bulletin 19: 11-15.

Drummond, A. J., M. A. Suchard, D. Xie, and A. Rambaut. 2012. Bayesian phylogenetics with BEAUti and the BEAST 1.7. Molecular Biology and Evolution 29: 1969-1973.

Edgar, R. C. 2004. MUSCLE: Multiple sequence alignment with high accuracy and high throughput. Nucleic Acids Research 32: 1792-1797.

Farris, J. S., M. Källersjö, A. G. Kluge, and C. Bult. 1995. Testing significance of incongruence. Cladistics 10: 315-319.

Felsenstein, J. 1981. Evolutionary trees from DNA sequences: A maximum likelihood approach. Journal of Molecular Evolution 17: 368-376.

Heled, J. and A. J. Drummond. 2010. Bayesian inference of species trees from multilocus data. Molecular Biology and Evolution 27: 570-580.

Huelsenbeck, J. P. and F. Ronquist. 2001. MrBayes: Bayesian inference of phylogeny. Bioinformatics 17: 754-755.

Hufford, L. 2003. Homology and developmental transformation: Models for the origins of the staminodes of Loasaceae subfamily Loasoideae. International Journal of Plant Sciences 164(Supplement): S409-S439.

Hufford, L., M. M. McMahon, A. M. Sherwood, G. Reeves, and M. W. Chase. 2003. The major clades of Loasaceae: Phylogenetic analysis using the plastid matK and trnL-trnF regions. American Journal of Botany 90: 1215-1228.

Hufford, L., J. J. Schenk, and J. M. Brokaw. 2016. Mentzelia. Pp. 496-543 in Flora of North America North of Mexico, vol. 12, ed. Flora North America Editorial Committee. New York: Oxford University Press.

Johnston, I. M. 1922. Undescribed plants mostly from Baja California University of California Publications in Botany 7: 437-446.

Johnston, I. M. 1924. Expedition of the California Academy of Sciences to the Gulf of California in 1921: The botany (the vascular plants). Proceedings of the California Academy of Science, $4^{\text {th }}$ Series 12: 951-1218.

Katoh, K. and D. M. Standley. 2013. MAFFT multiple sequence alignment software version 7: Improvements in performance and usability. Molecular Biology and Evolution 30: 772-780.

Kubatko, L. S. and J. H. Degnan. 2007. Inconsistency of phylogenetic estimates from concatenated data under coalescence. Systematic Biology 56: $17-24$

Lindell, J., F. R. Mendez-de la Cruz, and R. W. Murphy. 2005. Deep genealogical history without population differentiation: Discordance between mtDNA and allozyme divergence in the zebra-tailed lizard (Callisaurus draconoides). Molecular Phylogenetics and Evolution 36: 682-694.

Lira-Noriega, A., O. Toro-Núñez, J. R. Oaks, and M. E. Mort. 2015. The roles of history and ecology in chloroplast phylogeographic patterns of the bird-dispersed plant parasite Phoradendron californicum (Viscaceae) in the Sonoran Desert. American Journal of Botany 102: 149-164.

Liu, K., T. J. Warnow, M. T. Holder, S. M. Nelesen, J. Yu, A. P. Stamatakis, and C. R. Linder. 2012. SATé-II: Very fast and accurate simultaneous estimation of multiple sequence alignments and phylogenetic trees. Systematic Biology 61: 90-106.

Maddison, W. P. and D. R. Maddison. 2017. Mesquite: A modular system for evolutionary analysis. Version 3.31. http://mesquiteproject.org.

Marlowe, K. and L. Hufford. 2007. Taxonomy and biogeography of Gaillardia (Asteraceae): A phylogenetic analysis. Systematic Botany 32: 208-226.

Miller, M. A., W. Pfeiffer, and T. Schwartz. 2010. Creating the CIPRES Science Gateway for inference of large phylogenetic trees. Pp. 1-8 in
Proceedings of the Gateway Computing Environments Workshop (GCE). New Orleans: Gateway Computing.

Nason, J. D., J. L. Hamrick, and T. H. Fleming. 2002. Historical vicariance and postglacial colonization effects on the evolution of genetic structure in Lophocereus, a Sonoran Desert columnar cactus. Evolution 56: 2214-2226.

Nieto Feliner, G. and J. A. Rosselloì. 2007. Better the devil you know? Guidelines for insightful utilization of nrDNA ITS in species-level evolutionary studies in plants. Molecular Phylogenetics and Evolution 44: 911-919.

Nylander, J. A. A., J. C. Wilgenbusch, D. L. Warren, and D. L. Swofford. 2008. AWTY (are we there yet?): A system for graphical exploration of MCMC convergence in Bayesian phylogenetics. Bioinformatics 24: 581-583.

Pamilo, P. and M. Nei. 1988. Relationships between gene trees and species trees. Molecular Biology and Evolution 5: 568-583.

Paradis, E., J. Claude, and K. Strimmer. 2004. APE: Analyses of phylogenetics and evolution in R language. Bioinformatics 20: 289-290.

R Development Core Team. 2005. R: A language and environment for statistical computing. http:/ / cran.r-project.org.

Rambaut, A. and A. J. Drummond. 2005. Tracer v. 1.6. http:/ /beast.bio.ed.ac.uk/ Tracer.

Riddle, B. R., D. J. Hafner, and L. F. Alexander. 2000. Phylogeography and systematics of the Peromyscus eremicus species group and the historical biogeography of North American warm regional deserts. Molecular Phylogenetics and Evolution 17: 145-160.

Ronquist, F. and J. P. Huelsenbeck. 2003. MrBayes 3: Bayesian phylogenetic inference under mixed models. Bioinformatics 19: 1572-1574.

Saitou, N. and M. Nei. 1987. The neighbor-joining method: A new method for reconstructing phylogenetic trees. Molecular Biology and Evolution 4: $406-425$.

Schenk, J. J. and L. Hufford. 2011. Phylogeny and taxonomy of Mentzelia section Bartonia (Loasaceae). Systematic Botany 36: 711-720.

Schenk, J. J. 2013. Biogeographical diversification of Mentzelia section Bartonia in western North America. Journal of Biogeography 40: 455-465.

Shaw, J., E. B. Lickey, E. E. Schilling, and R. L. Small. 2007. Comparison of whole chloroplast genome sequences to choose noncoding regions for phylogenetic studies in angiosperms: The tortoise and the hare III. American Journal of Botany 94: 275-288.

Stamatakis, A. 2014. RAxML version 8: A tool for phylogenetic analysis and post-analysis of large phylogenies. Bioinformatics 30: 1312-1313.

Swofford, D. L. 2002. PAUP* Phylogenetic analysis using parsimony (*and other methods), v. 4.0. Sunderland: Sinauer Associates.

Taberlet, P., L. Gielly, G. Pautou, and J. Bouvet. 1991. Universal primers for amplification of three non-coding regions of chloroplast DNA. Plant Molecular Biology 17: 1105-1109.

Tamura, K. and M. Nei. 1993. Estimation of the number of nucleotide substitutions in the control region of mitochondrial DNA in humans and chimpanzees. Molecular Biology and Evolution 10: 512-526.

Tamura, K., M. Nei, and S. Kumar. 2004. Prospects for inferring very large phylogenies by using the neighbor-joining method. Proceedings of the National Academy of Sciences USA 101: 11030-11035.

Thiers, B. 2019. [continuously updated]. Index Herbariorum: A global directory of public herbaria and associated staff. New York Botanical Garden's Virtual Herbarium. http://sweetgum.nybg.org/science/ih/.

Thompson, H. J. 1963. Cytotaxonomic observations on Mentzelia, sect. Bartonia (Loasaceae). Madroño 17: 16-22.

Thompson, H. J. and J. E. Roberts. 1971. Observations on Mentzelia in southern California. Phytologia 21: 279-288.

Thompson, H. J. and J. E. Roberts. 1974. Loasaceae. Pp. 549-559 in A Flora of Southern California, ed. P. A. Munz. Berkeley: University of California Press.

Upton, D. E. and R. W. Murphy. 1997. Phylogeny of the side-blotched lizards (Phrynosomatidae:Uta) based on mtDNA sequences: Support for a midpeninsular seaway in Baja California. Molecular Phylogenetics and Evolution 8: 104-113.

Urban, I. and E. Gilg. 1900. Monographia Loasacearum. Nova Acta Leopoldina 76 : 1-370.

Wen, J. and E. Zimmer. 1996. Phylogeny and biogeography of Panax L. (the ginseng genus, Araliaceae): Inferences from ITS sequences of nuclear ribosomal DNA. Molecular Phylogenetics and Evolution 6: 167-177.

Weigend, M. 1997. Nasa and the Conquest of South America. Ph.D. dissertation. Munich, Bavaria: Ludwig-Maximilians-Universität.

Wilgenbusch, J. C., D. L. Warren, and D. L. Swofford. 2004. AWTY: A system for graphical exploration of MCMC convergence in Bayesian phylogenetic inference. http://king2.scs.fsu.edu/CEBProjects/awty/awty_start.php. 
Appendix 1. Voucher and GenBank information for taxa included in phylogenetic analyses. Listed as: taxon, collector and number, herbarium code, place of origin, latitude, longitude, and GenBank accession numbers (trnL-trnF, trnS-trnfM, ndhF-rpl32, rpl32-trnL, ITS, ETS). Accession information for outgroup taxa is listed as above. Herbarium acronyms follow Index Herbariorum (Thiers 2019). Accession numbers for new data begin with MG; an en-dash (-) indicates missing data.

Ingroup: M. hirsutissima, Angel 147, SD, California, San Diego Co., $32.9850^{\circ} \mathrm{N}, 116.3178^{\circ} \mathrm{W}, \mathrm{MG}^{26600,} \mathrm{MG726645,}$ MG726693, MG726548, MG726781, MG726735; M. hirsutissima, Boyd 3291, RSA, Baja California Sur, Mun. Mulegé, $27.5099^{\circ} \mathrm{N}, 114.308^{\circ} \mathrm{W}$, MG726618, MG726646, MG726705, MG726558, MG726795, MG726749; M. hirsutissima, Boyd 3309, RSA, Baja California Sur, Mun. Mulegé, $27.4746^{\circ} \mathrm{N}, 113.7203^{\circ} \mathrm{W}$, MG726601, MG726647, MG726694, MG726549, MG726782, MG726736; M. hirsutissima, Boyd 8088, RSA, Baja California Sur, Mun. Mulegé, $27.3490^{\circ} \mathrm{N}, 114.1666^{\circ} \mathrm{W}, \mathrm{MG}^{26620,}$ MG726648, MG726706, MG726559, MG726797, MG726750; M. hirsutissima, Breedlove 62325, CAS, Baja California Sur, Mun. Mulegé, 27.6028 $\mathrm{N}, 114.7749^{\circ} \mathrm{W}, \mathrm{MG726603,} \mathrm{MG726649,}$ —, MG726551, MG726791, MG726745; M. hirsutissima, Breedlove 62325, RSA, Baja California Sur, Mun. Mulegé, $27.6028^{\circ} \mathrm{N}, 114.7749^{\circ} \mathrm{W}$, MG726617, MG726650, MG726704, MG726566, MG726794, MG726748; M. hirsutissima, Breedlove 62415, CAS, Baja California Sur, Mun. Mulegé, $27.4900^{\circ} \mathrm{N}, 114.5484^{\circ} \mathrm{W}, \mathrm{MG} 726602, \mathrm{MG} 726651,-, \mathrm{MG} 726550, \mathrm{MG} 726790$, MG726744; M. hirsutissima, Clemons 1601, SD, Baja California, Mun. Mexicali, $32.3500^{\circ} \mathrm{N}, 115.8300^{\circ} \mathrm{W}$, MG726604, MG726652, MG726710, MG726567, MG726802, MG726755; M. hirsutissima, Clemons 1992, SD, Baja California, Mun. Mexicali, $32.1445^{\circ} \mathrm{N}, 115.6835^{\circ} \mathrm{W}, \mathrm{MG} 726605, \mathrm{MG} 726653$, MG726695, MG726552, MG726783, MG726737; M. hirsutissima, Clemons 2365, SD, Baja California, Mun. Mexicali, $32.4467^{\circ} \mathrm{N}, 115.8067^{\circ} \mathrm{W}$, MG726606, MG726664, MG726707, MG726568, MG726798, MG726751; M. hirsutissima, Fritsch 1289, RSA, Baja California, Mun. Ensenada, 28.9992 ${ }^{\circ} \mathrm{N}, 113.5746^{\circ} \mathrm{W}, \mathrm{MG726619}$ MG726654, - , MG726578, MG726796, MG726762; M. hirsutissima, Glacy 16, SD, California, San Diego Co., $32.8989^{\circ} \mathrm{N}, 116.2411^{\circ} \mathrm{W}, \mathrm{MG}^{26607,} \mathrm{MG} 726655, \mathrm{MG} 726708, \mathrm{MG} 726569$, MG726799, MG726752; M. hirsutissima, Hufford 2680, WS, Baja California Sur, Mun. Mulegé, $27.4719^{\circ} \mathrm{N}, 114.1013^{\circ} \mathrm{W}$, MG726608, MG726656, MG726696, MG726553, HM357377, HM440999; M. hirsutissima, Moran 12893, SD, Baja California, Mun. Ensenada, $29.4688^{\circ} \mathrm{N}, 113.5740^{\circ} \mathrm{W}$, MG726609, MG726657, MG726697, MG726554, MG726784, MG726738; M. hirsutissima, Moran 12916, CAS, Baja California, Mun. Ensenada, $29.4663^{\circ} \mathrm{N}, 113.5501^{\circ} \mathrm{W}, \mathrm{MG}^{26610}, \mathrm{MG}^{26658}, \mathrm{MG} 726698, \mathrm{MG} 726555$, MG726785, MG726739; M. hirsutissima, Moran 12939, RSA, Baja California, Mun. Ensenada, $29.5527^{\circ} \mathrm{N}, 113.5745^{\circ} \mathrm{W}$, MG726611, MG726659, MG726699, MG726556, MG726786, MG726740; M. hirsutissima, Moran 19767, SD, Baja California Sur, Mun. Mulegé, $27.2148^{\circ} \mathrm{N}, 114.3502^{\circ} \mathrm{W}$, MG726612, MG726660, - MG726561, MG726801, MG726754; M. hirsutissima, Moran 20008, SD, Baja California Sur, Mun. Mulegé, 27.3166 ${ }^{\circ} \mathrm{N}, 114.4344^{\circ} \mathrm{W}, \mathrm{MG}^{26613}, \mathrm{MG726661}$ MG726709, MG726560, MG726800, MG726753; M. hirsutissima, Moran 20231, SD, Baja California, Mun. Ensenada, $28.3614^{\circ} \mathrm{N}, 115.2053^{\circ} \mathrm{W}$, MG726614, MG726662, MG726700, MG726557, MG726787, MG726741; M. hirsutissima, Prigge 4605, RSA, Baja California, Mun. Ensenada, $30.0658^{\circ} \mathrm{N}, 115.7182^{\circ} \mathrm{W}$, MG726615, MG726663, MG726701, MG726564, MG726792, MG726746; M. hirsutissima, Thorne 61749, RSA, Baja California, Mun. Mexicali,

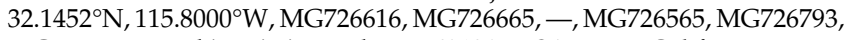
MG726747; M. hirsutissima, Thorne 63128, RSA, Baja California, Mun. Ensenada, $28.3472^{\circ} \mathrm{N}, 115.2195^{\circ} \mathrm{W}$, MG726621, MG726666, MG726702, MG726562, MG726788, MG726742; M. hirsutissima, Wisura 4858, RSA, Baja California, Mun. Ensenada, 29.9681 ${ }^{\circ}$, $115.4359^{\circ} \mathrm{W}, \mathrm{MG726622,}$ MG726667, MG726703, MG726563, MG726789, MG726743; M. involucrata var. involucrata, Fishbein 4520, WS, Sonora, Mun. San Luis Río Colorado, $32.0917^{\circ} \mathrm{N}, 114.2083^{\circ} \mathrm{W}$, MG726627, MG726672, MG726714, MG726573, MG726805, MG726758; M. involucrata var. involucrata, Gross 3007, RSA, California, San Bernardino Co., $35.0677^{\circ} \mathrm{N}, 116.6286^{\circ} \mathrm{W}, \mathrm{MG} 26628$, MG726673, MG726715, MG726574, MG726806, MG726759; M. involucrata var. involucrata, Hendrickson 572, SD, California, San Diego Co., $33.3056^{\circ} \mathrm{N}$, 116.0865W, MG726629, MG726674, MG726716, MG726575, MG726807, MG726760; M. involucrata var. involucrata, Hodgson and Mittleman s.n. 15 Apr 1979, WS, Arizona, Yuma Co., $33.3300^{\circ} \mathrm{N}, 113.9528^{\circ} \mathrm{W}$, FJ917852, FJ917760, FJ917943, MG726577, HM357385, HM441005; M. involucrata var. megalantha, Andre 6760, UCR, California, Riverside Co., $34.0607^{\circ} \mathrm{N}$,
116.4368, MG726624, MG726669, MG726712, MG726571, MG726803, MG726756; M. involucrata var. megalantha, Bell 305, SD, California, Imperial Co., $32.928^{\circ} \mathrm{N}, 114.5158^{\circ} \mathrm{W}$, MG726625, MG726670, MG726713, MG726572, MG726804, MG726757; M. involucrata var. megalantha, De Groot 4622, RSA, California, Riverside Co., $33.9139^{\circ} \mathrm{N}, 115.0772^{\circ} \mathrm{W}$, MG726626, MG726671, MG726718, MG726579, MG726809, MG726763; M. involucrata var. megalantha, Hill 33541, UCR, California, Riverside Co., $33.9508^{\circ} \mathrm{N}, 116.6389^{\circ} \mathrm{W}$, MG726630, MG726675, MG726717, MG726576, MG726808, MG726761; M. reflexa, Andre 7587 , UCR, California, Inyo Co., $35.9063^{\circ} \mathrm{N}, 116.6185^{\circ} \mathrm{W}$, MG726633, MG726678, MG726721, MG726583, MG726811, MG726764; M. reflexa, Brokaw 474, WS, California, San Bernardino Co., $35.7858^{\circ} \mathrm{N}$, 117.3625W, MG726634, MG726679, MG726722, MG726584, MG726812, MG726765; M. reflexa, Charlton 1684, RSA, California, Kern Co., $36.8701^{\circ} \mathrm{N}$, 117.9042 W, MG726635, MG726680, MG726724, MG726588, MG726815, MG726768; M. reflexa, Holmgren and Holmgren 14325, NY, Nevada, Nye Co., $36.8342^{\circ} \mathrm{N}, 116.6911^{\circ} \mathrm{W}, \mathrm{MG726636}$, MG726681, MG726723, MG726585, HM357439, HM441054; M. reflexa, Morefield 3431, RSA, California, Mono Co., $37.4859^{\circ} \mathrm{N}, 118.3219^{\circ} \mathrm{W},-$, MG726682, -, MG726586, MG726813, MG726766; M. reflexa, Morefield 3700, RSA, California, Mono Co., $37.4859^{\circ} \mathrm{N}, 118.3219^{\circ} \mathrm{W}, \mathrm{MG726637,}$ MG726683, - MG726587, MG726814, MG726767; M. tricuspis, Brokaw 065, WS, Arizona, Mohave Co., $36.0161^{\circ} \mathrm{N}$, 114.7372W, FJ917853, FJ917761，FJ917944， MG726591， MG726818, MG726769; M. tricuspis, Holmgren 14284, RSA, Nevada, Clark Co., $36.7919^{\circ} \mathrm{N}, 114.8644^{\circ} \mathrm{W}, \mathrm{MG726638}$ MG726685, MG726729, MG726594, MG726821, MG726772; M. tricuspis, Hufford 4005, WS, Nevada, Clark Co., $37.3150^{\circ} \mathrm{N}, 114.864^{\circ} \mathrm{W}$, MG726639, MG726686, MG726726, MG726592, MG726819, MG726770; M. tricuspis, Hufford 553, WS, Nevada, Clark Co., $36.1777^{\circ} \mathrm{N}, 114.7665^{\circ} \mathrm{W}, \mathrm{MG726640,}$ MG726687, MG726727, MG726593, HM357452, HM441067; M. tricuspis, Tiehm 14746, RSA, Nevada, Lincoln Co., $36.9063^{\circ} \mathrm{N}, 114.1875^{\circ} \mathrm{W}, \mathrm{MG726641,}$ MG726688, MG726728, MG726595, MG726820, MG726771; M. tridentata, Henrickson 16618, RSA, California, San Bernardino Co., $35.1366^{\circ} \mathrm{N}, 117.2615^{\circ} \mathrm{W}$, MG726642, MG726689, MG726730, MG726596, MG726822, MG726773; M. tridentata, Henrickson 16764, RSA, California, San Bernardino Co., $34.7581^{\circ} \mathrm{N}$, 116.8187ºW, MG726643, MG726690, MG726731, MG726597, MG726823, MG726774; M. tridentata, Henrickson 17965b, RSA, California, Inyo Co., $35.9881^{\circ} \mathrm{N}, 117.9227^{\circ} \mathrm{W}$, 一, MG726691, 一, MG726598, MG726824, MG726775.

Outgroup: M. affinis, Brokaw 211, WS, California, San Luis Obispo Co., $35.3514^{\circ} \mathrm{N}, \quad 119.9861^{\circ} \mathrm{W}, \quad F J 917854 ，$ FJ917762， FJ917945， MG726542, MG726776, —; M. aspera, Hufford 4022, WS, Peru, Depto. Amazonas, $5.6240^{\circ} \mathrm{S}, 78.4847^{\circ} \mathrm{W}, \mathrm{FJ} 917849$, FJ917757, FJ917940, MG726543, MG726777, MG726732; M. congesta, Brokaw 084, WS, California, Mono Co., $38.6383^{\circ} \mathrm{N}$, 119.5067²W, FJ917869, FJ917777, FJ917959, MG726544, MG726778, MG726733; M. desertorum, Brokaw 061, WS, California, Riverside Co., $33.8200^{\circ} \mathrm{N}, \quad 116.3894^{\circ} \mathrm{W}$, FJ917875, FJ917783, FJ917964, MG726545, MG726779, MG726734; M. dispersa, Brokaw 362, WS, California, Ventura Co., $34.7750^{\circ} \mathrm{N}, \quad 118.9681^{\circ} \mathrm{W}$, MG726599, MG726644, MG726692, MG726546, MG726780, —; M. eremophila, Brokaw 049, WS, California, Kern Co., $35.3771^{\circ} \mathrm{N}, 117.8659^{\circ} \mathrm{W}$, MN615137, FJ917969, FJ917882, FJ917790, 一, -; M. goodrichii, Hufford 4144, WS, Utah, Duchesne Co., $39.9622^{\circ} \mathrm{N}$, 110.4957 ${ }^{\circ}$ W, FJ917850, FJ917758, FJ917941, MG726547, HM357375, HM440998; M. humilis, Shenk 892, WS, New Mexico, Chavez Co.,

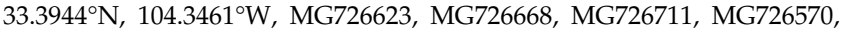
HM357379, HM441000; M. laevicaulis, Hitchcock 21852, WS, Washington, Whitman Co., $46.4354^{\circ} \mathrm{N}, 117.1540^{\circ} \mathrm{W}$ ，FJ917851，FJ917759，FJ917942, MG726580, HM357390, HM441008; M. marginata, Hufford 4339, WS, Colorado, Delta Co., $38.7464^{\circ} \mathrm{N}, 107.6686^{\circ} \mathrm{W}$, MG726631, MG726676, MG726719, MG726581, HM357397, HM441014; M. micrantha, Brokaw 266, WS, California, Ventura Co., $34.2020^{\circ} \mathrm{N}, 118.7779^{\circ} \mathrm{W}$, MN615138, FJ917984, FJ917897, FJ917805, -, -; M. nitens, Brokaw 079, WS, California, Mono Co., $37.5081^{\circ} \mathrm{N}, 118.5836^{\circ} \mathrm{W}, \mathrm{MN} 615139$, FJ918001, FJ917913, FJ917822, —, —; M. oligosperma, Schenk 918, WS, Texas, - , - MG726632, MG726677, MG726720, MG726582, MG726810, —; M. pectinata, Brokaw 053, WS, California, Kern Co., $35.0167^{\circ} \mathrm{N}, 119.4169^{\circ} \mathrm{W}$, MN615140, FJ918012, FJ917924, FJ917833, -, -; M. thompsonii, Brokaw 345, WS, New Mexico, San Juan Co., $36.7813^{\circ} \mathrm{N}, \quad 108.4832^{\circ} \mathrm{W}$, MN615141, FJ918022, FJ917934, FJ917843, - - - M. torreyi, Brokaw 126, WS, Idaho, Twin Falls Co., $42.5703^{\circ} \mathrm{N}, 114.6069^{\circ} \mathrm{W},-$, MG726684, MG726725, MG726589, MG726817, —; M. torreyi, Hufford 1923, WS, Idaho, —, —, FJ917848, FJ917756, FJ917939, MG726590, MG726816, - . 